\title{
Registro de larvas de Sebekia sp. (Pentastomida: Sebekidae) en peces de la cuenca media del río Usumacinta, Tabasco, México
}

\author{
Registration of larvae of Sebekia sp. (Pentastomida: Sebekidae) in fish of the \\ middle basin of the Usumacinta river, Tabasco, Mexico
}

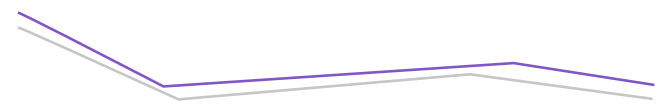

Raúl Enrique Hernández-Gómez*凶, Serapio López-Jiménez**, Martha Alicia Perera-García**, Carlos Alberto Cuenca-Soria*, Alfonso CastilloDomínguez*, Carlos Alfonso Álvarez González***, Carolina E. MelgarValdés*

\begin{abstract}
Hernández-Gómez, R. E., López-Jiménez, S., Perera-García, M. A., Cuenca-Soria, C. A., Castillo-Domínguez, A., Álvarez-González, C. A., \& Melgar-Valdés, C. E. (2018). Registro de larvas de Sebekia sp. (Pentastomida: Sebekidae) en peces de la cuenca media del río Usumacinta, Tabasco, México. Investigación y Ciencia de la Universidad Autónoma de Aguascalientes, 26(75), 5-12.
\end{abstract}

\section{RESUMEN}

Peces de importancia comercial en Tabasco, México, están siendo infestados de manera natural con parásitos de pentastómidos. En la presente investigación se dan a conocer la morfología, los índices ecológicos de infección de la larva en tres especies de valor comercial. Los peces bajo estudio son mojarras de interés alimenticio como Parachromis managuensis, Petenia splendida y Gobiomorus dormitor. Se registró un total de 30 larvas de pentastómidos. La mayor prevalencia registrada en estos fue en $\mathrm{G}$. dormitor de $30 \%$ y $35 \%$, durante los años 2005 y 2015, respectivamente. La prevalencia por larvas de pentastómido ha incrementado en las

Palabras clave: parásito; peces; morfología; prevalencia; intensidad, abundancia.

Keywords: parasite; fish; morphology; prevalence; intensity; abundance.

\section{Recibido: 9 de enero de 2018, aceptado: 30 de julio de 2018}

* División Académica Multidisciplinaria de los Ríos, Universidad Juárez Autónoma de Tabasco. Carretera Tenosique-Estapilla $\mathrm{Km}$. 1, Col. Solidaridad, C. P. 86901, Tabasco, México. Correo electrónico: h_raul_e@ hotmail.com; carlo.cuenca@ujat.mx; alfosin75@gmail.com; cemv81@ gmail.com

** División Académica de Ciencias Agropecuarias, Universidad Juárez Autónoma de Tabasco. Carretera Villahermosa-Teapa Km. 25+2, Ranchería la Huasteca 2da sección, C. P. 86298, Villahermosa Centro Tabasco, México. Correo electrónico: tabascotrema@hotmail.com; pereragarcia@hotmail.com

*** División Académica de Ciencias Biológicas, Universidad Juárez Autónoma de Tabasco. Carretera Villahermosa-Cárdenas $\mathrm{Km} .0 .5 \mathrm{~S} / \mathrm{N}$, Entronque a Bosques de Saloya, C. P. 86150, Tabasco, México. Correo electrónico: alvarez_alfonso@hotmail.com

$凶$ Autor para correspondencia especies estudiadas. Por tanto, se recomienda evitar el consumo de ejemplares crudos en diferentes platillos, y se propone al sector salud tomar las medidas precautorias ante la sociedad para evitar infección a humanos por esta larva.

\section{ABSTRAC1}

Commercially important fish in Tabasco, Mexico have been naturally infested with pentastomid parasite. The present research the morphology show information about the ecological indexes of infection of the larvae in three species of fish of commercial importance. The fish under study are mojarra, which are of alimentary interest such as Parachromis managuensis, Petenia splendida and Gobiomorus dormitor. A total of 30 pentastomid larvae were registered. The highest prevalence in these fish was in G. dormitor $30 \%$ and $35 \%$, during the year 2005 and 2015. The prevalence by pentastomid larvae has increased in the studied species, therefore, avoiding the consumption of raw fish in different dishes is recommended, and it is proposed to the health sector to establish caution measures in order to avoid infection to humans by this larvae.

\section{INTRODUCCIÓN}

Los pentastómidos que infectan a los peces causan daños relativamente menores a sus hospederos, reduciendo su crecimiento y productividad. Son parásitos obligados que se alimentan de mucosidad, 
IIVESTIGACIÓn Y CIERCIA DE LA UNIVERSIDAD AUTÓNOMA DE AGUASCALIERTES fluidos de los tejidos y sangre de su hospedero (Bunkley-Williams \& Williams, 1995). Las larvas de pentastómidos son patógenas, se encuentran generalmente envueltas por una membrana fibrosa delgada y muy resistente que los aísla de los tejidos del hospedero (Bunkley-Williams \& Williams, 1995). Morfológicamente, los pentastómidos poseen dos pares de ganchos desiguales localizados en la parte ventral anterior alrededor de la boca (Venard \& Bangham, 1941). Los adultos viven en el tracto respiratorio de reptiles considerados hospederos definitivos, que generalmente son serpientes, cocodrilos, lagartos y mamíferos (Riley, 1986).

Las aves y peces se infectan al ingerir el huevo en el agua o al consumir alimentos contaminados con materia fecal y/o mucosidad respiratoria de un animal que alberga pentastómidos adultos (Venard \& Bangham, 1941). Junker y Boomker (2002) registraron pentastómidos en cocodrilo, lagartos y tortugas infectados por vía natural, al ingerir peces parasitados, como Oreochromis mossambicus y Tilapia rendalli provenientes del Parque Nacional de Kruger, en el sur de África. Por otro lado, Bangham (1939) estudió a los parásitos de peces, en particular a la familia Centrarchidae sunfish en Florida, EE. UU., en donde registró el estado de larva de pentastómido.

En América Tropical y en especial México los pentastómidos son un grupo poco estudiado en cuanto a taxonomía y biología (Olson \& Cosgrove, 1982). Los humanos son considerados hospederos paraténicos, que se infestan de manera accidental y pueden albergar a la larva pentastómida; por ejemplo el Armillatus sp. y el Linguatula serrata, que pueden encontrarse enquistados en cápsulas calcáreas, de esta forma mueren pronto y usualmente ocasionan pocas molestias (Flynn, 1973; Sellier, Garin, Frija, Aubrey, \& Soyer, 2004).

García Magaña \& López Jiménez (2008) señalaron que los parásitos tienen una gran importancia desde el punto de vista sanitario, biológico y económico de las poblaciones piscícolas. En el estado de Tabasco, México, las investigaciones sobre larvas de pentastómidos infestando peces han sido escasas. En 1985 se realizó el primer registro de larvas infestando a bagres dulceacuícolas Ictalurus meridionalis del río San Pedro, Balancán (PinedaLópez \& García Magaña, 1991). Recientemente, en especies como Centropomus undecimallis y Gobiomorus dormitor en Pantanos de Centla, Tabasco
(García Magaña \& López Jiménez, 2008). La presente investigación tiene como objetivo dar a conocer la morfología e índices ecológicos de infección de larvas de pentastómidos en tres especies de peces de importancia comercial: Parachromis managuensis, Petenia splendida y $G$. dormitor como hospederos intermediarios en la zona de los ríos del estado de Tabasco, México.

\section{MATERIALES Y MÉTODOS}

\section{Área de estudio y muestras}

Los peces $(n=120)$ analizados fueron obtenidos de la pesca comercial proveniente de la laguna Canitzán $\left(17^{\circ} 20^{\prime} \mathrm{N}\right.$ y $\left.91^{\circ} 25^{\prime} \mathrm{W}\right)$ de la cuenca media del río Usumacinta en Tenosique, Tabasco, México. Se tomaron muestras de 20 organismos de cada especie estudiada, tanto para el año 2005 como para 2015, respectivamente. Las mismas fueron G. dormitor (guabina), P. managuensis (mojarra pinta) y $P$. splendida (tenguayaca) como posibles hospederos intermediarios. Entre los años 2005 y 2015 no hubo investigación al respecto, por lo que se retomaron los datos de 2005 para complementar los estudios recientes y realizar un comparativo. La búsqueda de las larvas de pentastómidos se basó en la metodología propuesta por Moravec (1994) bajo previa identificación de los hospederos, basada en sus características. Posteriormente se registraron las características morfométricas de cada especie: longitud estándar, longitud patrón y anchura del cuerpo, medidas con un ictiómetro de madera de $60 \mathrm{~cm}$. El peso fue obtenido con una balanza granataria OHAUS ${ }^{\circledR}$ de $2,610 \mathrm{~g}$ de capacidad.

\section{Diagnóstico de larvas de Sebekia sp.}

Se realizó una búsqueda bibliográfica sobre la clasificación taxonómica de la familia y del género Sebekidae para identificar la larva registrada en este estudio. Se realizó mediante la sistemática propuesta por Christoffersen y De Assis (2013); también se realizó la descripción morfológica de las mismas (Giesen, Takemoto, Calitz, Pérez Lizama, \& Junker, 2013; Venard \& Bangham, 1941). Para localizar las mismas los hospederos fueron eviscerados y fileteados con el objetivo de obtener piezas de musculatura de entre 5 y $8 \mathrm{~cm}$ de longitud, mismas que se colocaron en bolsas de polietileno de $20 \times 30 \mathrm{~cm}$, se etiquetaron con datos de colecta por hospedero para su posterior observación en menos de 24 h. Los filetes fueron comprimidos dentro de las bolsas de plástico con una prensa metálica. Se transparentaron en una 
caja de madera de $40 \times 40 \times 15 \mathrm{~cm}$ con cristal opaco y una lámpara de $30 \mathrm{~W}$ en su interior.

Las larvas encapsuladas de pentastómidos fueron localizadas con una lupa bifocal, extraídas con pinceles y agujas de disección finas, colocadas en cajas de Petri y observadas bajo un estereoscopio Zeiss ${ }^{\circledR}$. Posteriormente se fijaron con formaldehído al $4 \%$, se colocaron en frascos viales y se etiquetaron con los datos de colecta. Fueron temporalmente montados en un portaobjetos de $26 \times 76 \mathrm{~mm}$, con espesor de $\pm 1-1.2 \mathrm{~mm}$. Se estudiaron las estructuras externas e internas de cada larva, previamente aclaradas con Lactofenol de Amman (Lamothe-Argumedo, García-Prieto, Osorio-Sarabia, \& Pérez-Ponce de Léon, 1997). Después del aclarado las larvas fueron medidas con un ocular micrométrico ensamblado en un microscopio compuesto Lamobec ${ }^{\circledR}$.

Las mediciones se registraron en $\mathrm{mm}$ y fueron: longitud del cuerpo (LC), tomada desde el margen anterior hasta el margen posterior; anchura del cuerpo (AC), registrada en la parte media del cuerpo; longitud de la boca (LB) y anchura de la boca (AB). Todas las medidas anteriores fueron basadas en los criterios propuestos por Junker \& Boomker (2002). Se calcularon promedio y desviación estándar solo en larvas del año 2015, debido al número con que se contaba.

Para medir los ganchos de las larvas se siguió el esquema propuesto por Riley, Spratt y Winch (1990) y por Junker y Boomker (2002): (a-c) longitud de la hoja del gancho, (a-d) longitud total del gancho, (b-c) longitud de la base del gancho, (c-d) longitud horizontal del gancho, (c-e) longitud de espina accesoria y (F1-F2) punto de apoyo para el gancho. Algunas larvas fueron depositadas en la colección parasitológica de la División Académica de Ciencias Biológicas de la Universidad Juárez Autónoma de Tabasco con números de clave Guavina-Tenosique musculatura 22/03/05-Pt-004-001 y Pinta-Tenosiquehígado 21/03/05-Pt-004-003. Las larvas fueron fotografiadas con una cámara Cannon ${ }^{\circledR}$ ajustada al ocular del microscopio LABOMED CXRIII ${ }^{\circledR}$.

Los índices ecológicos se calcularon mediante la propuesta de Margolis, Esch, Holmes, Kuris y Schad (1982), fueron prevalencia (P), que indica el porcentaje de organismos parasitados por una especie de parásitos ( $\mathrm{P}=$ núm. hospederos infectados/núm. hospederos examinados x 100); intensidad media (IM), que es el promedio de especies de parásitos en organismos infectados (IM= núm. total de parásitos/ núm. hospederos infectados; así como la abundancia $(A B)$, que es el número de promedio de parásitos por hospederos examinados $(A B=$ núm. total de una especie de parásito/núm. hospederos examinados).

RESULTADOS

Se registraron 30 larvas de Sebekia sp. en 120 peces analizados, de las cuales seis se recolectaron en 2005 y 24 en el año 2015. Fueron localizadas microscópicamente en la musculatura de los peces. Generalmente presentaron un cuerpo alargado de coloración blanca, se distingue la parte anterior del mismo con cuatro ganchos y una espina accesoria en el margen superior de cada gancho. Los ganchos están ubicados a un lado de la boca, que es de forma oval. El cuerpo de estas larvas presentó anillos transversales con aspecto segmentado, en ellos se observaron pequeñas espinas finas. Debajo de cada anillo se observaron pequeñas muescas de forma circular. En la parte posterior del cuerpo presentaron una abertura que desemboca al exterior (ano). Esto se puede observar en la figura 1.

\section{Descripción morfométrica de las larvas de pentastómidos durante el año 2005}

Las larvas de pentastómidos ( $n=2)$ obtenidas de la mojarra tenguayaca $P$. splendida $(n=20)$ presentaron una longitud total del cuerpo de $3.01 \mathrm{~mm}$, con una anchura de $0.87 \mathrm{~mm}$, la boca presentó una longitud de $0.08 \mathrm{~mm}$ y su anchura fue $0.05 \mathrm{~mm}$. La distancia entre los anillos del cuerpo fue de $0.01 \mathrm{~mm}$. Las espinas del cuerpo tuvieron una longitud promedio de $0.01 \mathrm{~mm}$. En la guabina G. dormitor $(n=20)$, las larvas $(n=2)$ presentaron una longitud total del cuerpo de $4.5 \mathrm{~mm}$, con una anchura de $0.62 \mathrm{~mm}$. La boca presentó una longitud de $0.08 \mathrm{~mm}$ y una anchura de $0.04 \mathrm{~mm}$. La distancia entre los anillos del cuerpo fue de $0.08 \mathrm{~mm}$.

Las espinas del cuerpo tuvieron una longitud promedio de $0.09 \mathrm{~mm}$. En la mojarra pinta $P$. managuensis $(n=20)$ la longitud promedio total del cuerpo de las larvas $(n=2)$ fue de de $3.9 \mathrm{~mm}$, con una anchura de $0.61 \mathrm{~mm}$. La boca presentó una longitud de $0.08 \mathrm{~mm}$ y una anchura de $0.05 \mathrm{~mm}$. La distancia entre los anillos fue de $0.07 \mathrm{~mm}$. Las espinas presentaron una longitud promedio de 0.01 $\mathrm{mm}$. Todas las larvas tuvieron cuatro ganchos con 


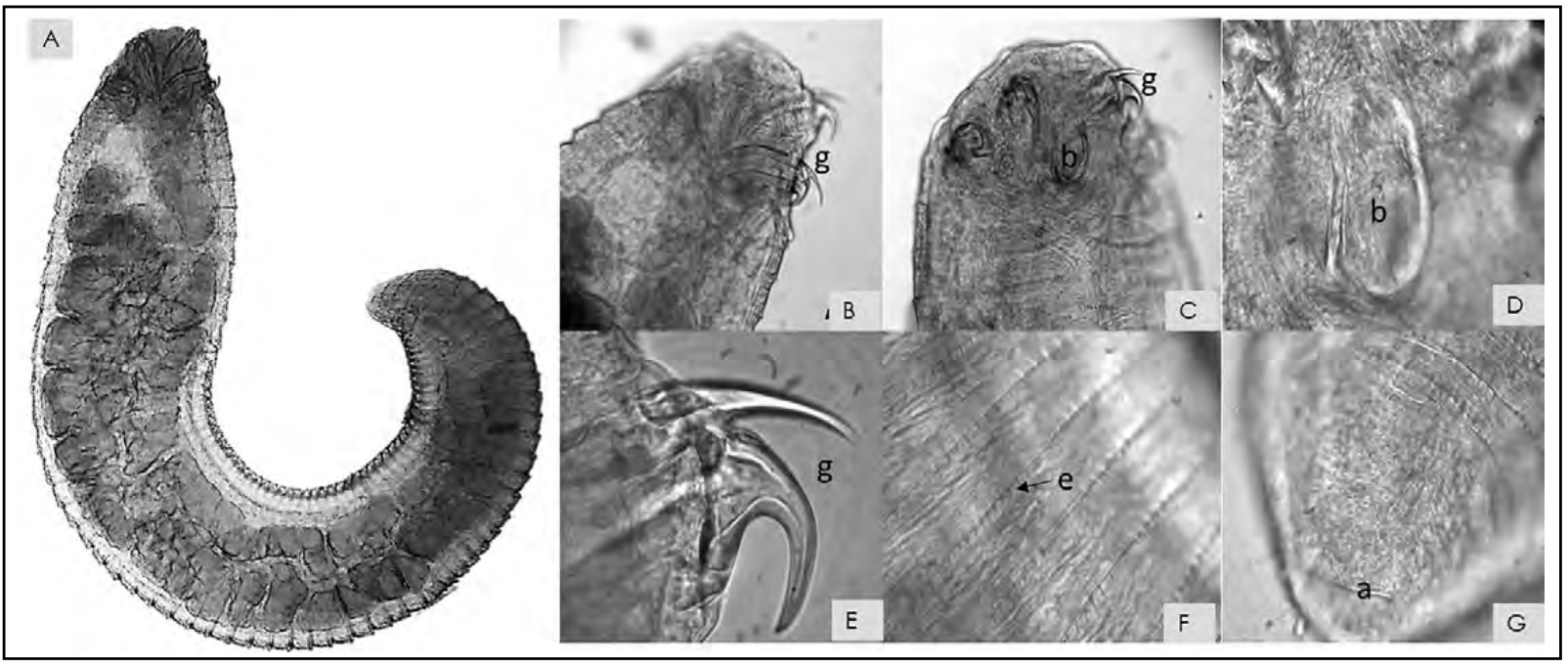

Figura 1. (A) Cuerpo de larvas; (B) vista dorsal del cuerpo, región anterior; (C) vista frontal del cuerpo, región anterior; (D) boca; (E) estructura de ganchos; (F) anillos con espinas; (G) vista posterior del cuerpo. (b): boca; (e): espinas; (g): ganchos dobles; (a): ano.

Fotografía del equipo de estudio.

una espina accesoria, además de 65-72 anillos en el cuerpo (tabla 1).

Descripción morfométrica de las larvas de pentastómidos durante el año 2015

Las larvas $(n=9)$ de pentastómidos registrada en $P$. splendida $(n=20)$ presentaron una longitud del cuerpo de 3.37 a $5.75 \mathrm{~mm}(4.76 \pm 0.743 \mathrm{~mm})$ con un ancho de 0.42 a $0.45(0.43 \pm 0.016 \mathrm{~mm})$. La boca presentó una longitud de 0.11 a $0.14 \mathrm{~mm}(0.12 \pm$ $0.055 \mathrm{~mm}$ ), su ancho fue de 0.062 a $0.087 \mathrm{~mm}(0.073$ $\pm 0.033 \mathrm{~mm}$ ). La distancia entre anillo de espinas fue de 0.01 a $0.12 \mathrm{~mm}(0.025 \pm 0.037 \mathrm{~mm})$. La longitud de la espina del cuerpo fue de 0.007 a 0.007 mm (0.007 \pm

Tabla 1

Medidas de los ganchos según Junker (2002)tomadas durante 2005

\begin{tabular}{ccccccc}
\hline & $\mathrm{a}-\mathrm{c}$ & $\mathrm{a}-\mathrm{d}$ & $\mathrm{b}-\mathrm{c}$ & $\mathrm{c}-\mathrm{e}$ & $\mathrm{c}-\mathrm{d}$ & $\mathrm{F}$ 1-F2 \\
\hline Larva $(\mathrm{n}=1) / P$. splendida & & & & & & \\
\hline $1^{\circ}$ gancho & 0.038 & 0.075 & 0.038 & 0.038 & 0.063 & 0.175 \\
$2^{\circ}$ gancho & 0.050 & 0.088 & 0.045 & 0.050 & 0.050 & 0.138 \\
$3^{\circ}$ gancho & 0.050 & 0.075 & 0.050 & 0.037 & 0.063 & 0.100 \\
$4^{\circ}$ gancho & 0.050 & 0.082 & 0.030 & 0.038 & 0.055 & 0.125 \\
\hline Larva $(\mathrm{n}=1) / G$. dormitor & & & & & & \\
\hline $1^{\circ}$ gancho & 0.080 & 0.100 & 0.055 & 0.043 & 0.038 & 0.162 \\
$2^{\circ}$ gancho & 0.062 & 0.088 & 0.050 & 0.037 & 0.063 & 0.200 \\
$3^{\circ}$ gancho & 0.062 & 0.100 & 0.062 & 0.050 & 0.050 & 0.125 \\
$4^{\circ}$ gancho & 0.063 & 0.088 & 0.062 & 0.050 & 0.038 & 0.100 \\
\hline Larva $(\mathrm{n}=1) / P$. managuensis & & & & & & \\
\hline $1^{\circ}$ gancho & 0.055 & 0.085 & 0.050 & 0.037 & 0.055 & 0.125 \\
$2^{\circ}$ gancho & 0.025 & 0.050 & 0.045 & 0.037 & 0.038 & 0.100 \\
$3^{\circ}$ gancho & 0.063 & 0.075 & 0.050 & 0.025 & 0.045 & 0.188 \\
$4^{\circ}$ gancho & 0.025 & 0.057 & 0050 & 0.025 & 0.038 & 0.163 \\
\hline
\end{tabular}

Nota: Elaboración propia. 


\section{IIVESTIGAGIÓn Y CUERCIA DE LA UกIVERSIDAD AUTÓOOOMA DE RGUASCHLIETTES}

$0.000 \mathrm{~mm}$ ). El cuerpo está formado de 59 a 78 anillos (tabla 2). En la mojarra pinta $P$. managuensis $(n=$ 20) se encontraron larvas $(n=3)$, que tuvieron una longitud del cuerpo de 3.8 a $4.5 \mathrm{~mm},(4.10 \pm 0.360$ $\mathrm{mm})$, ancho de cuerpo 0.42 a $0.52 \mathrm{~mm}(0.45 \pm 0.577$ $\mathrm{mm})$, longitud de la boca 0.10 a $0.12 \mathrm{~mm}(0.11 \pm 0.013$ $\mathrm{mm})$, ancho de boca 0.06 a 0.07 (0.07 $\pm 0.007 \mathrm{~mm}$ ), distancia entre anillo 0.01 (0.01 $\pm 0.0 \mathrm{~mm})$, longitud de la espina del cuerpo es $0.007 \mathrm{~mm}(0.007 \pm 0.007 \mathrm{~mm})$, el cuerpo está formado de 62 a 70 anillos (tabla 2).

En la guabina $G$. dormitor $(n=20)$ se obtuvieron las larvas $(n=12)$ de pentastómidos que presentaron una longitud de cuerpo entre 3.25 a $4.62 \mathrm{~mm}$ (3.89 $\pm 0.455 \mathrm{~mm})$, un ancho del cuerpo entre 1.15 y 1.37 $\mathrm{mm}$ (1.25 $\pm 0.077 \mathrm{~mm}$ ), la longitud de la boca de 0.07 a $1.35 \mathrm{~mm}(0.32 \pm 0.456 \mathrm{~mm})$, y ancho de boca de 0.05 a $1.35 \mathrm{~mm}(0.19 \pm 0.279 \mathrm{~mm})$, longitud de la espina del cuerpo fue de 0.007 a $0.009 \mathrm{~mm}(0.007 \pm$ $0.0075 \mathrm{~mm}$ ). El cuerpo está formado por entre 62 y 70 anillos (tabla 2).

\section{Índices ecológicos de infección}

Los índices de infección por las larvas de pentastómidos en los peces hospederos de importancia comercial registrados en 2005 fue mayor en la especie G. dormitor con una intensidad de 1.4;
0.35 de abundancia y $35 \%$ de prevalencia. Esto fue igual para 2015, con una intensidad de 2; 0.60 de abundancia y $30 \%$ de prevalencia. En las especies $P$. splendida y $P$. managuensis estos valores fueron bajos (tabla 3).

\section{DISCUSIÓN}

Las larvas de pentastómidos registradas en el presente estudio fueron encontradas encapsuladas en una membrana fibrosa en la musculatura de los peces estudiados durante 2005 y 2015, respectivamente. También presentaron características morfológicas y morfométricas similares. Fueron semejantes a las características anatómicas señaladas para las larvas de la familia Sebekidae (Barton \& Morgan, 2016; Bunkley-Williams \& Williams, 1995). Estas pueden llegar a medir hasta $7 \mathrm{~mm}$ de longitud, lo cual podría estar condicionado por la etapa de desarrollo de la especie (siete etapas) y/o a una variación intraespecífica (Bunkley-Williams \& Williams, 1995). Estas larvas son similares a la morfometría de la especie Sebekia oxicephala registrada en peces poecilidos como Phalloceros harpagos en Cambé, Paraná, Brasil, en peces C. undecimalis, I. puntatus y Dormitor maculatus de Florida, EE. UU., a las larvas registradas en pe-

Tabla 2

Medidas de los ganchos según Junker (2002)tomadas durante 2015

\begin{tabular}{lcccccc}
\hline & $a-c$ & $a-d$ & $b-c$ & $c-e$ & $c-d$ & F1-F2 \\
\hline Larvas ( $n=9$ )/P. splendida & & & & & & \\
\hline $1^{\circ}$ gancho & 0.033 & 0.054 & 0.083 & 0.056 & 0.036 & 0.124 \\
$2^{\circ}$ gancho & 0.037 & 0.062 & 0.188 & 0.062 & 0.041 & 0.158 \\
$3^{\circ}$ gancho & 0.088 & 0.058 & 0.139 & 0.069 & 0.038 & 0.147 \\
$4^{\circ}$ gancho & 0.037 & 0.058 & 0.138 & 0.062 & 0.040 & 0.156 \\
\hline Larvas (n= 12)/G. dormitor & & & & & & \\
\hline $1^{\circ}$ gancho & 0.06 & 0.087 & 0.048 & 0.085 & 0.048 & 0.145 \\
$2^{\circ}$ gancho & 0.062 & 0.082 & 0.049 & 0.084 & 0.049 & 0.152 \\
$3^{\circ}$ gancho & 0.058 & 0.076 & 0.047 & 0.073 & 0.044 & 0.145 \\
$4^{\circ}$ gancho & 0.057 & 0.077 & 0.046 & 0.074 & 0.044 & 0.142 \\
\hline Larvas (n=3) en P. managuensis & & & & & & \\
\hline $1^{\circ}$ gancho & 0.051 & 0.075 & 0.045 & 0.095 & 0.054 & 0.141 \\
$2^{\circ}$ gancho & 0.05 & 0.079 & 0.044 & 0.075 & 0.054 & 0.133 \\
$3^{\circ}$ gancho & 0.05 & 0.079 & 0.049 & 0.069 & 0.051 & 0.147 \\
$4^{\circ}$ gancho & 0.06 & 0.066 & 0.075 & 0.077 & 0.05 & 0.113 \\
\hline
\end{tabular}

Nota: Elaboración propia. 
Tabla 3

Índices ecológicos de infección según hospedero

\begin{tabular}{|c|c|c|c|c|c|c|}
\hline \multirow{3}{*}{ Índices/año } & \multicolumn{6}{|c|}{ Hospedero } \\
\hline & \multicolumn{2}{|c|}{ P. splendida } & \multicolumn{2}{|c|}{ G. dormitor } & \multicolumn{2}{|c|}{ P. managuensis } \\
\hline & 2005 & 2015 & 2005 & 2015 & 2005 & 2015 \\
\hline Intensidad & 1.00 & 1.80 & 1.40 & 2.00 & 1.00 & 3.00 \\
\hline Abundancia & 0.05 & 0.45 & 0.35 & 0.60 & 0.10 & 0.15 \\
\hline Prevalencia & 5.00 & 25.00 & 35.00 & 30.00 & 10.00 & 5.00 \\
\hline Número de larvas & 1.00 & 9.00 & 7.00 & 12 & 2 & 3 \\
\hline Peces parasitados & 1 & 5 & 5 & 6 & 2 & 1 \\
\hline Peces revisados & 20 & 20 & 20 & 20 & 20 & 20 \\
\hline
\end{tabular}

Nota: Elaboración propia.

ces cíclidos del parque nacional Kruger, África (Almeida, Silva-Souza, \& Sales, 2010; Junker, Boomker, \& Booyse, 1998; Venard \& Bangham, 1941), también concuerdan con los datos morfométricos de las larvas de Sebekia sp. registradas en el pez bobo liso I. meridionalis, en el robalo C. undecimalis y en guabina G. dormitor en Tabasco, México (García-Magaña \& López-Jiménez, 2008; Pineda-López \& García-Magaña, 1991).

Sin embargo, Salgado-Maldonado, PinedaLópez, Vidal-Martínez y Kennedy (1997) registraron larvas de $S$. subtriquetra en Laguna Vapor de Campeche, México; en peces de la familia Cichlidae como Cichlasoma synspilum y Cichlasoma urophthalmus, las cuales presentan caracteres morfológicos similares a las del presente estudio, pero difieren en cuanto al número de anillos del cuerpo de las larvas. Fueron diferentes a las larvas Sebekia wedli, Subtriquetra rileyi, L. gracile, Subtriqueta sp. 1 y Subtriqueta sp. 2, en sus caracteres morfológicos y morfométricos; principalmente en el número de anillos en el cuerpo (Junker et al., 1998; Vicentin et al., 2013).

Las larvas de este estudio fueron diferentes a las de otras especies de pentastómido, como Subtriquetra subtiquetra, L. serrata, Almelifer sp., Lingutala sp., Reighardia stemea y Alofia merki, principalmente por el tamaño del cuerpo, ganchos y espinas accesorias (Barton \& Morgan, 2016; Lazo et al., 1999; Martínez et al., 2004; Vargas, 1971).

Con respecto a los índices ecológicos registrados en el presente estudio por larvas de Sebekia sp., el hospedero G. dormitor fue quien registró los valores máximos de intensidad y prevalencia en ambos años de investigación. Asimismo, $P$. splendida mostró similares resultados, lo que indica que la abundancia y prevalencia de esta larva aumentó. En P. managuensis, los índices se presentaron con poca variación si se comparan con el registro de las dos especies señaladas anteriormente. Estos se asemejan a la vez a los valores obtenidos en los hospederos C. undecimalis y G. dormitor (García Magaña \& López Jiménez, 2008).

Sin embargo, difieren de los índices señalados en el bagre I. meridionalis del río San Pedro en Balancán, Tabasco, México (Pineda López \& García Magaña, 1991). También difieren de las prevalencias de $9.2 \%$ y $40.5 \%$ registradas en peces cíclidos con larvas de S. wedli y en peces poecílidos infestados con S. oxycephala. Lo mismo con peces carácidos infestados con larvas de Sebekia sp. en Brasil (Almeida et al., 2010; Giesen et al., 2013; Junker et al., 1998). También presentaron diferencia con las señaladas en Protonibea diacanthus (Sciaenidae) con prevalencias entre $0.4 \%$ y $33 \%$, infestados con larvas de A. merki, y con las prevalencias de $6 \%$ con larvas de Sebekia purdieae en peces de Australia (Barton \& Morgan, 2016).

CONCLUSIONES

Con base en los análisis realizados a los caracteres morfológicos y morfométricos de las larvas de pentastómido del presente estudio, se establece que la larva que infesta los peces en Tabasco, México, corresponde a la especie S. oxycephala. Sin 


\section{IIVESTIGAGIÓn Y CUERCIA DE LA UחIVERSIDAD AUTÓNOMA DE AGUASCALIERTES}

embargo, deben realizarse estudios más exhaustivos (moleculares) para la confirmación de la especie. Por otra parte, se propone a las autoridades sanitarias del sector acuícola y del sector salud realicen medidas precautorias para prevenir una zoonosis debida a estos parásitos por el consumo de peces insuficientemente cocidos. Las enfermedades por pentastómidos en otros países son pentastomiasis visceral, causada por el parásito en forma de larva, y pentastomiasis respiratoria, causada por parásitos adultos (Christoffersen \& De Assis, 2015).

REFERENCIAS

- Almeida, W. O., Silva-Souza, A. T., \& Sales, D. L. (2010). Parasitism of Phalloceros harpagos (Cyprinodontiformes: Poeciliidae) by Sebekia oxycephala (Pentastomida: Sebekidae) in the headwaters of the Cambé River, Paraná State, Brazil. Brazilian Journal of Biology, 70(2), 457-458.

- Andersson, R. C. (1995). F. Moravec: Parasitic nematodes of freshwater fishes of Europe. Folia Parasitologica, 42 (3), 240.

- Bangham, R. U. (1939). Parasites of Centrarchidae from Southern Florida. Transactions of the American Fisheries Society, 68(1), 263-268.

- Barton, D. P., \& Morgan, J. A. T. (2016). A morphological and genetic description of pentastomid infective nymphs belonging to the family Sebekidae Sambom, 1922 in fish in Australian waters. Folia Parasitologica, 63: 026. doi: 10.14411/ fp. 2016.026

- Bunkley-Williams, L., \& Williams, E. H. (1995). Parásitos de peces de valor recreativo en agua dulce de Puerto Rico. Lajas, Puerto Rico, Puerto Rico: Universidad de Puerto Rico.

- Christoffersen, M. L., \& De Assis, J. E. (2013). A systematic monograph of the recent Pentasomida, with a compilation of their hosts. Zoologische Mededelingen, 87(1), 1-206.

(2015). Pentastomida. Revista IDE@-SEA, 98B, 1-10. Recuperado de http://sea-entomologia.org/IDE@/ revista_98A.pdf

- Flynn, R. J. (1973). Parasites of laboratory animals. Ames, lowa, US: State University Press.

- García Magaña, L., \& López Jiménez, S. (2008). Parásitos de peces de la reserva de la biósfera "Pantanos de Centla", Tabasco: Algunas recomendaciones para su prevención y control. Revista de Divulgación Kuxulkab, 14 (26), 13-22.

- Giesen, S. C., Takemoto, R. M., Calitz, F., Pérez Lizama, M. A., \& Junker K. (2013). Infective pentastomid larvae from Pygocentrus nattereri Kner (Pisces, Characidae) from the Miranda River, Pantanal, Mato Grosso do Sul State, Brazil, with notes on their taxonomy and epidemiology. Folia Parasitologica, 60(5), 457468.

- Junker, K., \& Boomker, J. (2002). Description of Pelonia africana n. g., n. sp. (Pentastomida: Sebekidae) from the lungs of Pelomedusa subrufa and Pelusios sinuatus (Chelonia) in South
Africa. Onderstepoort Journal of Veterinary Research, 69, 5359

- Junker, K., Boomker, J., \& Booyse, D. G. (1998). Experimental studies on the life-cycle of Sebekia wedli (Pentastomida: Sebekidae). Onderstepoort Journal of Veterinary Research, 65(4), 233-237.

- Lamothe-Argumedo, R., García-Prieto, L., Osorio-Sarabia, D., \& Pérez-Ponce de Léon, G. (1997). Catálogo de la Colección Nacional de Helmintos. México, D. F.: Universidad Nacional Autónoma de México-CONABIO

- Lazo, R. F., Hidalgo, E., Lazo, J. E., Bermeo, A., Llaguno, M., Murillo, J., \& Teixeira, V. P. (1999). Ocular linguatuliasis in Ecuador: Case report and morphometric study of the larva of Linguatula serrata. The American Journal of Tropical Medicine and Hygiene, 60(3), 405-409.

- Margolis, L., Esch, G. W., Holmes, J. C., Kuris, A. M., \& Schad, G. M. (1982). The use of ecological terms in parasitology (Report of an ad hoc committee of the American Society of Parasitologists). The Journal of Parasitology, 68(1), 131-133.

- Martínez, J., Criado-Fornelio, A., Lanzarot, P., FernandezGarcía, M., Rodríguez-Caabeiro, F., \& Merino, S. (2004). A new pentastomid from the black vulture. Journal of Parasitology. 90(5), 1103-1105.

- Moravec, F. (1994). Parasitic nematodes of freshwater fishes of Europe (473 pp.). Prague-Dordrecht-Boston-London: Academia and Kluwer Academic Publishers.

- Olson, A. C., \& Cosgrove, G. E. (1982). Pentastomida. En S. Hurlbert, \& A. Villalobos-Figueroa (Eds.), Aquatic biota of Mexico, Central America and the West Indies. US: San Diego State University Press.

- Pineda López, R., \& García Magaña, L. (1991). Hallazgo de ninfas de pentastómidos en Ictalurus meridionalis (Pisces: Ictaluridae) en el Río San Pedro, Balancán, Tabasco. Nota de investigación. Universidad y Ciencia, 8(16), 97-100.

- Riley, J. (1986). The biology of pentastomids. Advances in Parasitology, 25, 45-128. Spratt, D. M., \& Winch, J. M. (1990). A revision of the genus Sebekia Sambon, 1922 (Pentastomida) from crocodilians with descriptions of five new species. Systematic Parasitology, 16(1), 1-25. 
- Salgado-Maldonado, G., Pineda-López, R., Vidal-Martínez, V. M., \& Kennedy, C. R. (1997). A checklist of metazoan parasites of cichlid fish from Mexico. Journal of the Helminthological Society of Washington, 64 (2), 195-207.

- Sellier, P., Garin, Y. J., Frija, J., Aubrey, A., \& Soyer, P. (2004). Multiple thoracoabdominal calcifications in a healthy west african man. Clinical Infectious Diseases. An Official Publication on the Infectious Diseases Society of America, 39(10), 14751476. doi: $10.1086 / 425362$

- Vargas, V. M. (1971). Hallazgo de Subtriqueta subtriqueta Sambon, 1922 (Pentastomida) en Costa Rica y su redescripción. Revista Latinoamericana de Microbiología, 13,137-146.

- Venard., C. E., \& Bangham, R. V. (1941). Sebekia oxycephala (Pentastomida) from Florida Fishes and Some Notes on the Morphology of the Larvae. Ohio Journal of Science, 41 (1), 23-28.

- Vicentin, W., Ibarrola Vieiria, K. R., Roland Tavares, L. E., Dos Santos Costa, F. E., Massato Takemoto, R., \& Paiva, F. (2013). Metazoan endoparasites of Pygocentrus nattereri (Characiformes: Serrasalminae) in the Negro River, Pantanal, Brazil. The Brazilian Journal of Veterinary Parasitology, Jaboticabal, 22 (3), 331-338.Valera, estado Trujillo, Venezuela. Boletín de Malariología y Salud Ambiental, 47(2), 56-59. 